\title{
Recent studies of heavy ion transfer reactions using large solid angle spec- trometers
}

\author{
Suzana Szilner ${ }^{1, *}$, Lorenzo Corradi ${ }^{2}$, Giovanni Pollarolo ${ }^{3}$, Giulia Colucci $^{4}$, Petra Colović $^{1}$, Enrico Fioretto ${ }^{2}$, Franco \\ Galtarossa $^{2}$, Alain Goasduff ${ }^{2,4}$, Tommaso Marchi ${ }^{2}$, Tea Mijatović ${ }^{1}$, Giovanna Montagnoli ${ }^{4}$, Daniele Montanari ${ }^{2}$, Fer- \\ nando Scarlassara ${ }^{4}$, Alberto M. Stefanini ${ }^{2}$, and Javier Jose Valiente-Dobón ${ }^{2}$
}

${ }^{1}$ Ruđer Bošković Institute, Zagreb, Croatia

${ }^{2}$ INFN - Laboratori Nazionali di Legnaro, Legnaro, Italy

${ }^{3}$ Dipartimento di Fisica, Università di Torino, and Istituto Nazionale di Fisica Nucleare, Torino, Italy

${ }^{4}$ Dipartimento di Fisica, Università di Padova, and Istituto Nazionale di Fisica Nucleare, Padova, Italy

\begin{abstract}
We present selected results recently obtained in the study of heavy ion transfer reactions at energies close to the Coulomb barrier by employing the large solid angle magnetic spectrometer PRISMA. We discuss the production of neutron-rich heavy nuclei via multinucleon transfer processes and the related effects of secondary processes, in particular nucleon evaporation, studied in a high resolution kinematic coincidence experiment. We also present the recent results in the studies of neutron-neutron correlations for closed shell and superfluid systems.
\end{abstract}

\section{Introduction}

Nucleon transfer reactions at energies close to the Coulomb barrier always played an important role for nuclear structure and reaction dynamics. In the studies of the reaction mechanism, it was important to define the evolution of the reaction mechanism from the quasi-elastic regime to the more complex deep-inelastic and fusion [1]. For energies close to the Coulomb barrier, transfers constitute the largest part of the total reaction cross section and are the main source for the energy dissipation from the relative motion to the intrinsic excitation. With heavyions multiple transfer of nucleons becomes available in the reaction giving the possibility to study the relative role of single particle and pair transfer modes $[2,3]$.

The exchange of nucleons in multinucleon transfer reactions has been recently used as a suitable mechanism to populate moderately neutron-rich nuclei. Nuclear reaction models predict large primary multinucleon transfer cross sections $[4,5]$ that are comparable, or even larger, than those of other reactions like fragmentation at intermediate energies or low-energy fusion with radioactive beams. Being the transfer processes governed by form factors and optimum Q-value considerations, with neutron deficient projectiles on heavy targets only proton-stripping and neutron pick-up channels, for the projectiles, are available, while with neutron-rich projectiles also proton pickup and neutron stripping channels, for the projectiles, open up. This corresponds, for the heavy partner, to the population in the south-east direction, leading to the neutronrich heavy region, which is presently receiving peculiar

*e-mail: szilner@irb.hr attention. Due to the characteristic behavior of the binding energy, the process is essentially governed by the lighter partner of the reaction.

In order to employ multinucleon transfer reactions for studying both the production of neutron rich nuclei and the effects of the residual interaction, one needs to reach good mass and charge resolution, as well as high efficiency to measure low cross sections for massive transfer channels. All these demanding requirements are fulfilled by large solid angle spectrometers based on trajectory reconstruction [6-8]. The coupling of these spectrometers with large $\gamma$ arrays [9-11] allowed, in addition, to identify $\gamma$ rays coming from the decay of weak transfer channels associated with the population of nuclei moderately far from stability.

\section{Generals on detection techniques}

Different techniques have been employed to identify nuclei produced in transfer reactions. Most of these techniques make use of magnetic spectrographs or spectrometers for a complete identification of nuclear charge, mass and energy of final reaction products (see Ref. [1] and references therein). Magnetic spectrographs were mostly used in light ion transfer reactions, where, by combining magnetic elements of different complexity to focus momenta, it was possible to distinguish the excited states. With heavier ions more demanding conditions are required in order to keep a good resolution and to have at the same time a sufficient detection efficiency, given the large energy dynamic range of transfer products. For these ions, time-of-flight spectrometers have been designed (see for 


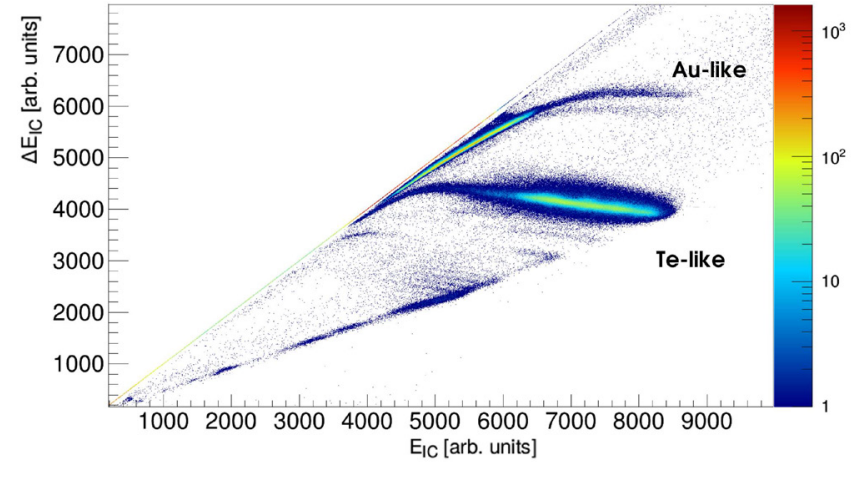

Figure 1. Matrix of $\Delta E$ versus $E$ measured in the PRISMA ionization chamber (IC) for the ${ }^{197} \mathrm{Au}+{ }^{130} \mathrm{Te}$ reaction at $E_{\text {lab }}\left({ }^{197} \mathrm{Au}\right)=1.07 \mathrm{GeV}$ and $\theta_{\text {lab }}=37^{\circ}$. The most intense band corresponds to Te-like ions, while Au-like ions are located in the region where $\Delta E_{\mathrm{IC}}$ and $E_{\mathrm{IC}}$ merge. One can also see the ions corresponding to fission-like events which are not stopped in the IC.

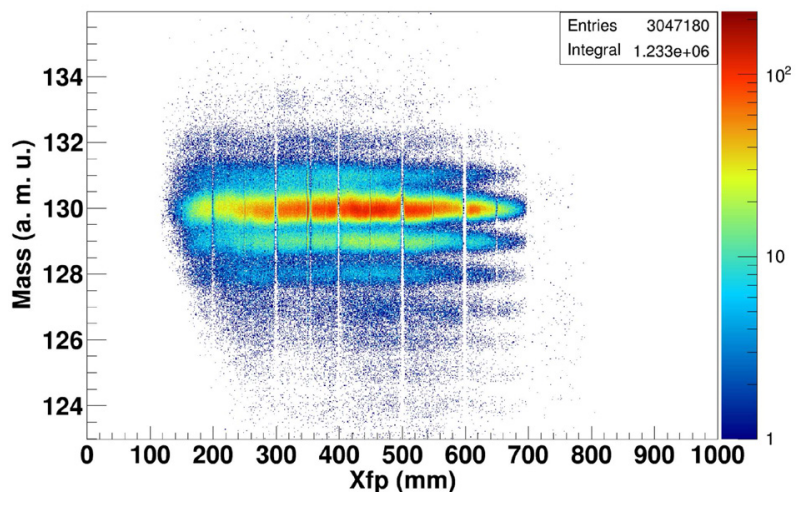

Figure 2. Mass vs position in the focal plane (Xfp) obtained in the ${ }^{197} \mathrm{Au}+{ }^{130} \mathrm{Te}$ [20] at $E_{\text {lab }}=1.07 \mathrm{GeV}$ after the trajectory reconstruction and software-based correction of nonlinear distortions of ion trajectories in PRISMA for the Te-like ions.

example Refs. [12-14] and references therein) providing a good $\mathrm{A}$ and $\mathrm{Z}$ resolution for medium mass ions. These spectrographs or spectrometers have solid angles in the range 3-10 msr. Beyond these values, it becomes unfeasible to use complex magnetic elements to correct for the ion optical aberrations. The solution for the large solid angle spectrometers ( $\sim 100 \mathrm{msr})$ was to simplify the magnetic element configuration and to apply the concept of trajectory reconstruction $[6,15]$, by using a detector system which, besides nuclear charge, energy and timing, provides the necessary position information along the ion path. With these devices [6, 7], mass and nuclear charge identification has been successfully demonstrated for ions up to A 100-130, especially when using inverse kinematics conditions. We here briefly list the main characteristics of the PRISMA spectrometer and its detector system. A position-sensitive micro-channel plate detector [16] is placed at the entrance of the spectrometer, providing a start signal for time-of-flight measurements and bi-dimensional position signals. Ions pass through the optical elements of the spectrometer (a quadrupole and a dipole) and enter a focal plane detector [17] which is made of a parallel plate detector of multiwire type, providing timing and bi-dimensional position signals with resolutions similar to the entrance detector. Located at the end of the focal plane is an array of transverse field multiparametric ionization chambers (IC), providing nuclear charge via energy loss $(\Delta \mathrm{E})$ and total energy (E) measurements. The large dynamical acceptance of the IC is illustrated in Fig. 1 which shows the $\Delta E$ versus $E$ matrix for the ${ }^{197} \mathrm{Au}+{ }^{130} \mathrm{Te}$ reaction. In this figure, one observes the intense band corresponding to the Te-like ions (the spectrometer conditions were set for the detection of these events), as well as the $\mathrm{Au}$-like and fission-like events.

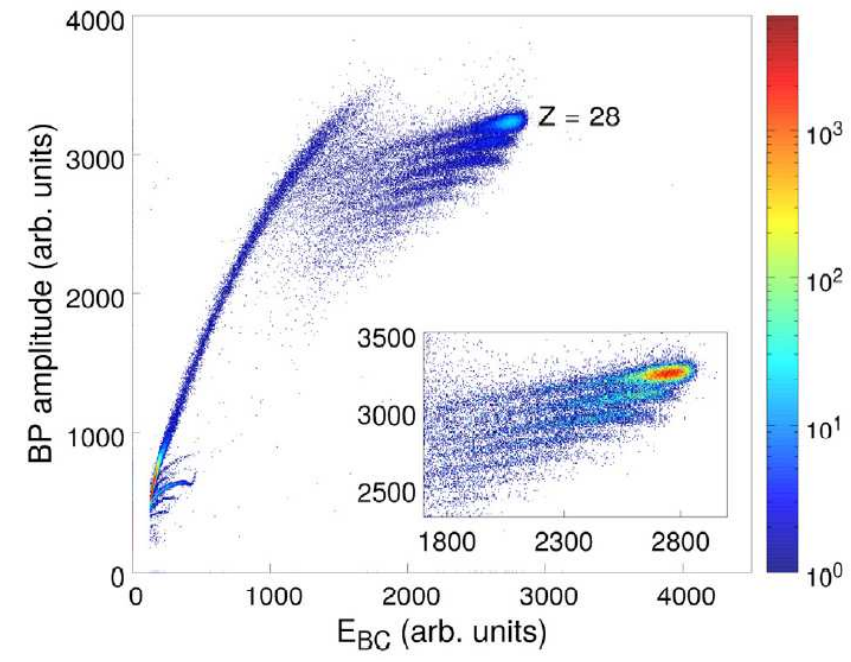

Figure 3. Two-dimensional scatter plots BP amplitude vs energy obtained for the reaction ${ }^{58} \mathrm{Ni}+{ }^{208} \mathrm{~Pb}$ at $E_{\text {lab }}\left({ }^{58} \mathrm{Ni}\right)=400 \mathrm{MeV}$ and $\theta_{\mathrm{lab}}=65^{\circ}$ measured in the Bragg ionization chamber of the coincident detector (NOSE) [21].

The described detector system gives all the necessary information for the complete ion identification, which is performed via an event-by-event reconstruction of the trajectory inside the magnetic elements $[6,18,19]$. The result of this procedure is depicted as an example in Fig. 2, demonstrating the quality of the mass separation [20].

For the detection of the heavy partner of the reaction a dedicated set-up [21] was specifically built and coupled to PRISMA. This allowed, via a high-resolution massmass correlation, to study the final mass distribution of the heavy partner and the effect of secondary processes. The coincident detector (NOSE), providing information on the heavy partner of the reaction, consists of a multiwire parallel-plate avalanche counter followed by an axialfield ionization chamber (Bragg chamber) and with a geometrical solid angle about one third of PRISMA. To illustrate the resolving power of this detector, we plot in Fig. 3 the Bragg peak (BP) amplitude vs energy obtained for the ${ }^{58} \mathrm{Ni}+{ }^{208} \mathrm{~Pb}$ reaction. This coincident detection of binary reaction fragments is very important in the context of un- 
derstanding the production mechanism of heavy neutronrich nuclei in regions of astrophysical interest, where secondary processes play a significant role.

The studies of transfer reactions at low energies greatly benefited from the use of large angle spectrometers, in particular, at energies below the barrier, where the transfer cross sections drop significantly. By employing inverse kinematics and by detecting ions at very forward angles, one has, at the same time, enough kinetic energy of the outgoing recoils (for energy and therefore mass resolution) and forward-focused angular distribution (high efficiency). This technique was utilized with the PRISMA spectrometer in the studies of the nucleon-nucleon correlations (see section 4) [22, 23].

\section{The synthesis of heavy neutron-rich nuclei}

The production of heavy neutron-rich nuclei in the region close to the $\mathrm{N}=126$ shell closure recently received a boost of interest being the properties of these nuclei fundamental for the understanding of the synthesis of heavy elements. The investigation of the nuclei in this region of the nuclear landscape is hampered by the difficulties in their production, as well as their direct identification with present detection techniques.

One of the promising mechanisms for the production of the heavy neutron-rich nuclei, as was already discussed in the Introduction, is nucleon transfer reactions between heavy ions at energies close to the Coulomb barrier [4]. The path leading to primary neutron-rich heavy partners, in multinucleon transfer reactions, has been investigated in recent experiments with neutron-rich stable projectiles, in ${ }^{64} \mathrm{Ni}+{ }^{238} \mathrm{U}$ [24], ${ }^{40} \mathrm{Ar}+{ }^{208} \mathrm{~Pb}$ [25], ${ }^{136} \mathrm{Xe}+{ }^{198} \mathrm{Pt} \mathrm{[26]} \mathrm{and}$ ${ }^{136} \mathrm{Xe}+{ }^{238} \mathrm{U}$ [27]. In these reactions the light partner has been identified directly via high-resolution magnetic spectrometers while, in some of the mentioned cases, information on the heavy partner was obtained indirectly by detecting the coincident $\gamma$ rays produced by the fragments. In these experiments information on secondary processes, i.e. neutron evaporation, could also be extracted as illustrated for example in Refs. [6, 28, 29]. In all these experiments it has been emphasized that secondary processes may significantly influence the final yield distributions, even though rather meager evidence of the survival probability of the heavy partner has been provided.

In order to understand and quantify the production process also for the heavy partner of the reaction, we performed an experiment with a simultaneous detection of light and heavy transfer products in the ${ }^{197} \mathrm{Au}+{ }^{130} \mathrm{Te}$ system [20]. We chose the neutron-rich ${ }^{130} \mathrm{Te}$ to populate neutron transfer channels leading primarily to neutron-rich $\mathrm{Au}$ isotopes. We exploited the performance of the PRISMA spectrometer [6] to identify isotopes in the tellurium region, while the coincident Au-like partners were detected with a dedicated set-up, NOSE [21], coupled to PRISMA. Favorable experimental conditions were achieved by employing inverse kinematics in such a way that both the light recoil and the heavy partner could have enough energy for their detection. With this set-up we were able to select binary events, Au-like events in NOSE detector were uniquely associated to the Te-like events detected in PRISMA.

For the mass identification of the reaction products, the reconstruction of their trajectory in the magnetic elements of PRISMA has been performed. The outcome of this procedure is the final mass distribution for the Te isotopes depicted in Fig. 4 (right panel) where the resolution turned out to be $\triangle A / A \sim 1 / 240$, allowing to separate the different isotopes. The obtained mass distribution in the coincident NOSE detector is shown in Fig. 4 (left panel), where the peaks are well centered at mass 197 and 130, corresponding to Au-like and Te-like ions, respectively. The mass resolution turned out to be $\triangle A / A \sim 1 / 44$, in agreement with the angle and timing resolutions of the used detectors. As already discussed, the NOSE detector was optimized for $\mathrm{Au}$-like in coincidence with Te-like ions in the spectrometer. One notices that the distribution is not symmetric around mass 197, but it displays a tail towards larger values. Part of the asymmetry may be due to a transfer mechanism as well as to experimental effects (e.g. non linear response of detectors or electronics) mimicking such tails. It is only when one sets a coincidence with a high resolution spectrometer that one can construct a mass-mass correlation matrix and infer about the behaviour of the heavy partner. This is nicely demonstrated in Fig. 5, where the reconstructed mass of the Au-like ions is correlated with that of the high resolution mass distribution of the Te-like ions detected in PRISMA.

In Fig. 5 we see that the identification in mass of the light fragment with high resolution allows to separate the mass distribution of the heavy partner in well-defined bands. The black dots indicate the centroids of the projections of each band, showing how they slightly bend toward lower masses in comparison to those expected for the corresponding primary neutron transfer channels (red circles). This indicates that the primary fragments acquire significant excitation energy so that evaporation becomes relevant in defining the final yields. This analysis allowed to extract information on the average number of evaporated neutrons for each channel associated with the Te isotopes. The effect of the evaporation depends strongly on the bombarding energy and on the projectile and target combination.

A successful theoretical description of the measured transfer cross sections was achieved with the model GRAZING [30-32], which includes surface phonons for the inelastic part and a collection of single particle levels for the treatment of nucleon exchange (see for example Refs. [25, 26] and references therein). Recently, the data were also compared with other models, for example with the Time-Dependent Hartree-Fock theory [33] which has been generalized in order to be able to calculate cross sections for transfer channels [34-37]. One of the critical point in these comparisons is the accurate treatment of the secondary process. Thus, from the experimental point of view it is necessary to provide the evidence of the survival probability of the heavy partner in high-resolution measurements where the light and heavy transfer products are simultaneously detected. From the theoretical point of 

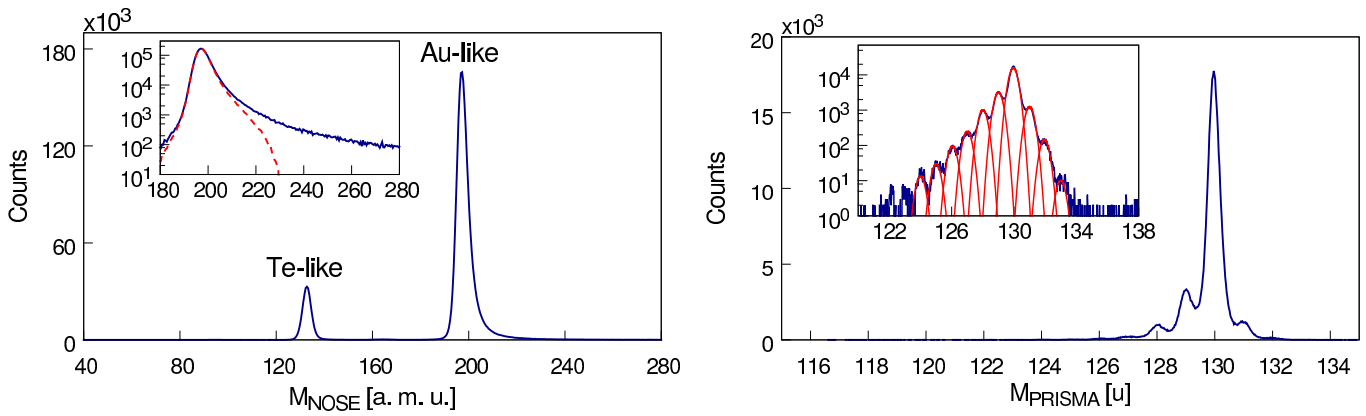

Figure 4. (left) Mass spectrum obtained employing the NOSE detector. The inset shows the mass distribution of Au-like events in logarithmic scale, with red-dashed curve with the coincidence of Te-like ions detected in PRISMA. (right) Mass distribution for the Te isotopes obtained after ion trajectory reconstruction in PRISMA. The inset shows the distribution in logarithmic scale with the multi-Gaussian fit used to evaluate the yields of the neutron transfer channels.

view, it is important to develop a fully microscopic model that incorporates the structure of the two reactants and the reaction dynamics on the same footing.

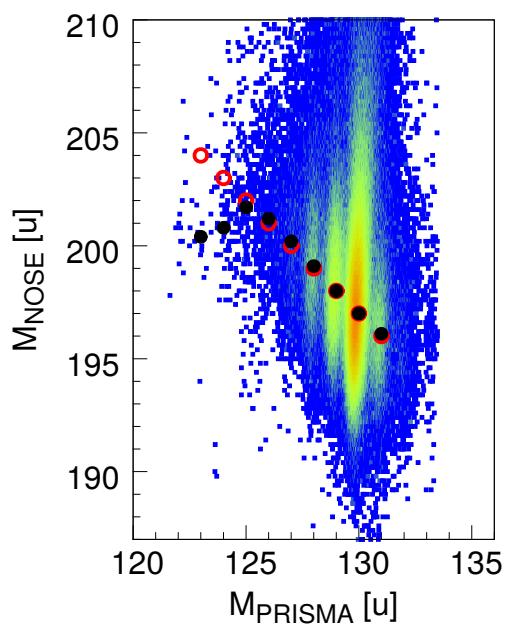

Figure 5. Mass-mass correlation matrix of Te isotopes detected in PRISMA and the heavy partner detected in coincident NOSE detector. The red (empty) circles indicate the centroids of the correlated masses of the primary neutron transfer channels, the black (full) dots indicate the experimental centroids as derived from the fits of their projections.

\section{Probing nucleon-nucleon correlations in transfer reactions}

Two-particle transfer reactions are one of the best probes of pairing correlations in nuclear systems. In the studies of such reactions the comparison between the cross section for two-particle transfer channels with the predictions for an independent nucleon transfer process should provide a direct measurement of nucleon-nucleon correlations. In order to study these correlations we recently measured the transfer probabilities for one- and two-nucleon transfer channels in heavy ion collisions over a wide energy range with cross sections spanning several orders of magnitude. In table 1 we list the systems measured so far with PRISMA, employing inverse or direct kinematics technique. For some of the indicates systems, PRISMA was coupled to a $\gamma$ array to measure the transfer strength to specific states.

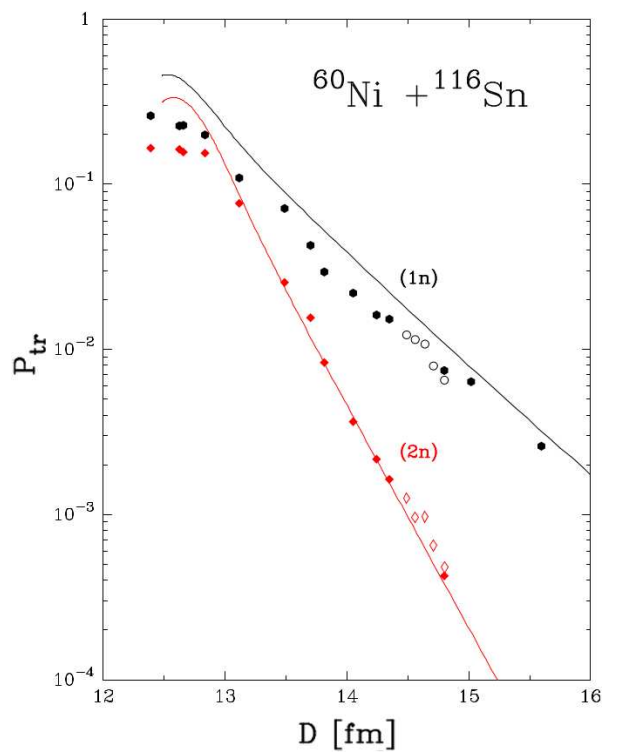

Figure 6. Experimental (points) and microscopically calculated (lines) transfer probabilities $\left(P_{\mathrm{tr}}\right)$ as a function of the distance of closest approach $(D)$ for one- $\left({ }^{61} \mathrm{Ni}\right)$ and two-neutron $\left({ }^{62} \mathrm{Ni}\right)$ transfers in the ${ }^{60} \mathrm{Ni}+{ }^{116} \mathrm{Sn}$ system. Open symbols correspond to the probabilities obtained from angular distribution in direct kinematics [38] while solid symbols refer to the excitation function measurement performed in inverse kinematics [23].

In the measured reactions, ${ }^{96} \mathrm{Zr}+{ }^{40} \mathrm{Ca}$ [22] and ${ }^{116} \mathrm{Sn}+{ }^{60} \mathrm{Ni}[23]$, (lighter) target recoils have been detected with PRISMA, and an excitation functions at several bombarding energies have been obtained from the Coulomb barrier to $20-25 \%$ below, reaching $\sim 15.5 \mathrm{fm}$ of distance of closest approach. It is convenient to extract the transfer probability $P_{\mathrm{tr}}$, defined as the ratio of the transfer cross section to the corresponding Rutherford cross section, and to plot these probabilities as a function of the distance of closest approach $D$ for a Coulomb trajectory. The extrac- 
Table 1. Systems studied with PRISMA, in stand-alone or coupled to a $\gamma$ array, employing inverse or direct kinematics technique.

\begin{tabular}{llll}
\hline system & item & set-up & Ref. \\
\hline${ }^{96} \mathrm{Zr}+{ }^{40} \mathrm{Ca}$ & excitation function, inverse kinematic & PRISMA & {$[22]$} \\
${ }^{116} \mathrm{Sn}+{ }^{60} \mathrm{Ni}$ & excitation function, inverse kinematic & PRISMA & {$[23]$} \\
& angular distribution, direct kinematic & PRISMA + AGATA & {$[38]$} \\
${ }^{206} \mathrm{~Pb}+{ }^{18} \mathrm{Sn}$ & excitation function, inverse kinematic & PRISMA & {$[39]$} \\
${ }^{92} \mathrm{Mo}+{ }^{54} \mathrm{Fe}$ & excitation function, inverse kinematic & PRISMA & {$[40]$} \\
& angular distribution, direct kinematic & PRISMA + $\mathrm{LaBr}_{3}$-array & {$[40]$} \\
\hline
\end{tabular}

tion of the transfer probability $P_{\operatorname{tr}}$ as a function of $D$ is rather alluring because the angular distributions obtained at different bombarding energies will be all superimposed, if the $Q$-value window does not change significantly. Further, the transfer probability at large distances $D$ may be obtained by measuring an excitation function at fixed center of mass angle or by measuring angular distributions at fixed energy (see Fig. 6).

The transfer probabilities for the mentioned two systems have been analyzed by employing a microscopic theory, which, for the first time in a heavy-ion collision, provided a consistent description of one- and two-neutron transfer channels, in shape and magnitude. In this microscopic theory to compute the inclusive one-neutron cross section we calculated the transfer probability for a given single particle transition and we obtained the total transfer probability by summing over all possible transitions that can be constructed from the single particle states in projectile and target. For the two-particle transfer we just mention that we diagonalized the total Hamiltonian with a model space containing only two-particle configuration coupled to $0^{+}$(i.e. transfer of a $\mathrm{J}=0^{+}$pair). Calculations reproduce well the experimental slope as well as the absolute values of the transfer probabilities for the one neutron channel. In the well Q-value matched system ${ }^{60} \mathrm{Ni}+{ }^{116} \mathrm{Sn}$, with the ground-to-ground state $Q$-value for one- and two-neutron transfers very close to the optimum, the microscopically calculated transfer probabilities well reproduce the experimental ones for $(2 n)$ channel (see Fig. 6 and Ref. [23] for details). We wish to emphasize that to calculate the $(2 n)$ channel one has to solve the well-known system of semiclassical coupled equations up to second-order Born approximation, whose amplitude consists of the simultaneous transfer of the pair of nucleons, the nonorthogonality term, and the term which represents the successive process via an intermediate channel. The ground states have been described in the BCS approximation with a standard state-independent pairing force. The nice agreement between data and calculations indicates that the two-neutron transfer channel in this system is populating essentially only the ground state, in agreement with the experimental total kinetic-energy loss distributions and consistently with particle- $\gamma$ measurements [38] which provided an upper limit of $24 \%$ for the possible excited state population. The nucleon-nucleon correlations induced by the pairing interaction, incorporated in the microscopic theory, were essential for the description of two-neutron transfers.
Recently, we also measured the probability for neutron transfer channels in the ${ }^{206} \mathrm{~Pb}+{ }^{118} \mathrm{Sn}$ reaction [39]. The main purpose of this measurement was to investigate how the effect of nucleon-nucleon correlations, in the evolution of the reaction, is modified in collisions between very heavy ions. The measurement was performed in inverse kinematics by using the newly developed ${ }^{206} \mathrm{~Pb}$ beam at superconducting PIAVE-ALPI accelerators of Laboratori Nazionali di Legnaro. This is the heaviest (asymmetric) semi-magic system (see table 1) with closed proton and open neutron shells, very well Q-value matched for neutron transfers. In the collisions of very heavy ions, the population of final states with high excitation energies and large angular momenta may significantly change the transfer strength of the ground-to-ground state transitions.

Besides neutron-neutron correlations, it is also important to study proton-proton and proton-neutron correlations, for which existing data are extremely scarce. To this purpose we measured the ${ }^{92} \mathrm{Mo}+{ }^{54} \mathrm{Fe}$ reaction where a nearly symmetric population of both neutron and proton pick-up and stripping channels are present. We refer to Ref. [40] for details. By using the large solid angle spectrometer PRISMA we were able to measure, beside the neutron transfer channels, also the proton transfers up to the large distances of closest approach, where the nuclear absorption is negligible.

\section{Summary and outlook}

The advent of the large solid angle magnetic spectrometers ensured significant advances in the field of multinucleon transfer reactions at energies close to the Coulomb barrier. The mechanism of the production of neutron rich heavy nuclei was studied in a high resolution kinematic coincidence in the ${ }^{197} \mathrm{Au}+{ }^{130} \mathrm{Te}$ system, where the extracted mass-mass correlations allowed to study the effect of secondary processes. These kind of research, besides the better understanding the transfer mechanism in itself, are of particular relevance in the optimization of multinucleon transfer mechanism for the population of neutron-rich nuclei.

The pairing correlations were discussed via transfer probabilities for one- and two-nucleon transfer channels. These probabilities have been compared with a microscopic theory that incorporates nucleon-nucleon correlation in the ${ }^{40} \mathrm{Ca}+{ }^{96} \mathrm{Zr}$ and ${ }^{60} \mathrm{Ni}+{ }^{116} \mathrm{Sn}$ systems. It is very important to continue these studies, and to compare the experimental observables with microscopic calculations where nucleon-nucleon correlations are treated together 
with the relevant structure and dynamics information. The issue connected with the pair correlations is presently at the focus of a renewal of interest [41-50], in particular in ongoing research with radioactive beams, where the pairing interaction may be significantly modified in nuclei with extended neutron distributions.

\section{Acknowledgments}

The material presented in this paper is the result of the work of many people who participated to different measurements and experimental preparation, whom we would like to thank. The authors acknowledge financial support from the European Regional Development Fund for the Center of Excellence for Advanced Materials and Sensing Devices (Grant No. KK.01.1.1.01.0001). This work was also partly supported by the Croatian Science Foundation under Project No. 7194 and in part under project no. IP2018-01-1257. The authors acknowledge financial support from HORIZON2020, ENSAR2 project (No. 654002).

\section{References}

[1] L. Corradi, G. Pollarolo, and S. Szilner, J. of Phys. G: Nucl. Part. Phys. 36, 113101 (2009).

[2] D. M. Brink and R. A. Broglia, Nuclear Superfluidity: Pairing in Finite Systems, Cambridge University Press (2005).

[3] R. A. Broglia and V. Zelevinsky, Fifty Years of Nuclear BCS - Pairing in Finite Systems, World Scientific (2013).

[4] C. H. Dasso, G. Pollarolo, and A. Winther Phys. Rev. Lett. 73, 1907 (1994).

[5] V. Zagrebaev and W. Greiner, Phys. Rev. Lett. 101, 122701 (2008).

[6] S. Szilner et al., Phys. Rev. C 76, 024604 (2007).

[7] S. Pullanhiotan et al., Nucl. Instrum. Methods Phys. Res. A 593, 343 (2008).

[8] F. Cappuzzello et al., Eur. Phys. J. A 52, 167 (2016).

[9] A. Gadea et al., Eur. Phys. J. A 20, 193 (2004).

[10] J. Simpson et al., Acta Physica Hungarica N.S. 11, 159 (2000).

[11] A. Gadea et al., Nucl. Instrum. Methods Phys. Res. A 654, 88 (2011); E. Farnea et al., Nucl. Instrum. Methods Phys. Res. A 621, 331 (2010); S. Akkoyun et al., Nucl. Instrum. Methods Phys. Res. A 668, 26 (2012).

[12] G. Montagnoli et al., Nucl. Instrum. Methods Phys. Res. A 454, 306 (2000).

[13] L. Corradi et al., Phys. Rev. C 66, 024606 (2002).

[14] S. Szilner et al., Phys. Rev. C 71, 044610 (2005).

[15] L. Corradi et al., Nucl. Instrum. Methods Phys. Res. B 317, 743 (2013).
[16] G. Montagnoli et al., Nucl. Instrum. Methods Phys. Res. A 547, 455 (2005).

[17] S. Beghini et al., Nucl. Instrum. Methods Phys. Res. A 551, 364 (2005).

[18] D. Montanari et al., Eur. Phys. J. A 47, 4 (2011).

[19] T. Mijatović et al., Eur. Phys. J. A 52, 113 (2016).

[20] F. Galtarossa et al., Phys. Rev. C 97, 054606 (2018).

[21] E. Fioretto et al., Nucl. Instrum. and Methods Phys. Res. A 899, 73 (2018).

[22] L. Corradi et al, Phys. Rev. C 84, 034603 (2011).

[23] D. Montanari et al., Phys. Rev. Lett. 113, 052501 (2014).

[24] L. Corradi et al., Phys. Rev. C 59, 261 (1999).

[25] T. Mijatović et al., Phys. Rev. C 94, 064616 (2016).

[26] Y. X. Watanabe et al., Phys. Rev. Lett. 115, 172503 (2015).

[27] A. Vogt et al., Phys. Rev. C 92, 024619 (2015).

[28] P. R. John et al., Phys. Rev. C 90, 021301(R) (2014).

[29] P. R. John et al., Phys. Rev. C 95, 064321 (2017).

[30] A. Winther, Nucl. Phys. A 572, 191 (1994).

[31] A. Winther, Nucl. Phys. A 594, 203 (1995).

[32] [http:/www.to.infn.it/ nanni/grazing], program GRAZING.

[33] C. Simenel and A. S. Umar, Progress in Part. and Nucl. Phys. 103, 19 (2018).

[34] C. Simenel, Phys. Rev. Lett. 105, 192701 (2010).

[35] K. Sekizawa and K. Yabana, Phys. Rev. C 88, 014614 (2013).

[36] K. Sekizawa and K. Yabana, Phys. Rev. C 93, 054616 (2016).

[37] A. V. Karpov and V. V. Saiko, Phys. Rev. C 96, 024618 (2017).

[38] D. Montanari et al., Phys. Rev. C 93, 054623 (2016).

[39] L. Corradi et al., JPS Conference Proceedings, in press.

[40] T. Mijatović et al., EPJ Web of Conferences in press.

[41] M. Evers et al., Phys. Rev. C 84, 054614 (2011).

[42] A. Lemasson et al., Phys. Lett. B 697, 454 (2011).

[43] G. Potel et al., Phys. Rev. Lett. 107, 092501 (2011).

[44] R. A. Broglia et al., Phys. Scr. 91, 063012 (2016).

[45] G. Scamps and D. Lacroix, Phys. Rev. C 87, 014605 (2013).

[46] G. Scamps and K. Hagino, Phys. Rev. C 92, 064602 (2015).

[47] G. Scamps et al., Phys. Rev. C 94, 064606 (2016).

[48] P. Magierski, K. Sekizawa, and G. Wlazlowski, Phys. Rev. Lett. 119, 042501 (2017).

[49] J. Dobaczewski et al., Phys. Rev. Lett. 72, 981 (1994).

[50] F. Barranco, G. Potel, E. Vigezzi, and R.A. Broglia, Eur. Phys. J. A 55, 104 (2019). 